\title{
Characterizing the mechanism of extracellular electron transfer from marine sulfur oxidizing microbes using high throughput genetic techniques.
}

Rowe, A. R. ${ }^{1}$, Kamble, N. ${ }^{1}$, Nealson, K. H. ${ }^{2}$ \& El-

Naggar M. Y. ${ }^{2}$

${ }^{1}$ Univeristy of Cincinnati, Cincinnati $\mathrm{OH}$ annette.rowe@uc.edu;

${ }^{2}$ University of Southern California, Los Angeles CA knealson@usc.edu; mnaggar@usc.edu

Little is known about the prevalence of mineral oxidation processes, though they are likely occurring in many environments. This stems, from our limited insight into the biochemistry of these metabolisms, especial utilizing solid mineral phases. To this aim, we have developed electrochemical cultivation techniques, to target enrichment and isolation of microbes capable of oxidative extracellular electron transfer (EET). Using these techniques in a marine sediment (Catalina Harbor, USA) we isolated a variety of electrode oxidizing microbes from the genera Thioclava, Marinobacter, Halomonas, Idiomarina, Thalassospira, and Pseudamonas; organisms commonly detected in marine sediments but not generally associated with mineral, sulfur and/or iron oxidation. Several of these isolates are facultatively heterotrophic, and we have recently developed genetic techniques that allow us to introduce a wide range of genetic mutations into their genomes. This work will present an in depth electrochemical characterization of the EET modes for several of these strains. We will also discuss insights from their genomes, as well as present preliminary results from high-throughput genetic screens, highlighting genes that confer a selective advantage under elemental sulfur and/or electrode oxidation relative to heterotrophic growth, as well a work pointing to elemental sulphur as a major source/sink for electrons in this sediment. 